\title{
Seguimiento fármaco-terapéutico en pacientes ambulatorios con tratamiento anti-retroviral
}

\author{
Yanneth Moya, Fernando Bernal, Eliana Rojas y Elizabeth Barthel
}

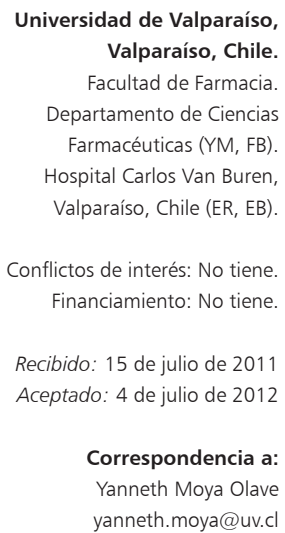

Conflictos de interés: No tiene. Financiamiento: No tiene.

Recibido: 15 de julio de 2011 Aceptado: 4 de julio de 2012

Correspondencia a: Yanneth Moya Olave yanneth.moya@uv.cl

\begin{abstract}
Pharmaceutical follow up of antirretroviral treatment in outpatients
Adherence to antiretroviral therapy is key contributor to decreasesing morbidity and mortality from HIV/ AIDS infection. However, it is affected by treatment-related factors including the multiple adverse reactions and interactions arising from chronic polypharmacy. In order to determine drug-related problems, 66 outpatients from Hospital Carlos Van Buren on antiretroviral therapy were monitored. $100 \%$ had medication-related problems and $46.1 \%$ of those problems were related to the safety of the therapy. Hypertriglyceridemia associated to the combined use of both nucleoside reverse transcriptase inhibitor and a non-nucleoside reverse transcriptase inhibitor was the most frequent adverse reaction. Results show that pharmacological monitoring of patients on antiretroviral treatment is necessary for the early identification of drug-related problems and for the proposal of alternatives that promote therapeutic safety and efficacy.

Key words: Pharmaceutical care, pharmacist-managed, HIV medication.

Palabras clave: Seguimiento farmacoterapéutico, tratamiento antirretroviral.
\end{abstract}

\section{Introducción}

L a terapia anti-retroviral (TARV) ha contribuido a controlar el desarrollo de la enfermedad por VIH/SIDA y mejorar la calidad de vida de los individuos infectados, reduciendo su morbilidad y mortalidad $^{1,2,3}$. Sin embargo, su eficacia terapéutica depende de la obtención de una adecuada adherencia al tratamiento prescrito ${ }^{4}$; la no adherencia favorece la posibilidad de desarrollo de subtipos de virus resistentes a los fármacos anti-retrovirales ${ }^{5}$. Entre los factores que influyen en la falta de adherencia se consideran los relacionados al paciente (edad, escolaridad, factores psicológicos y socioeconómicos), a la medicación (esquemas de dosificación, efectos adversos del TARV y polifarmacia prescrita), y al grado de conocimiento de la enfermedad y del tratamiento $^{4,6-8}$. Existe evidencia que señala a los efectos adversos causados por los anti-retrovirales como la principal causa de falta de adherencia al tratamiento ${ }^{6,9}$.

Se ha señalado que $96 \%$ de los pacientes que reciben TARV presentan -al menos- un medicamento que podría generar problemas de toxicidad o inefectividad de la terapia ${ }^{10}$. La identificación, prevención y manejo de las reacciones adversas se considera un aspecto de importancia en la obtención de los objetivos terapéuticos ${ }^{11,12}$.

En Chile existen 21 fármacos anti-retrovirales para el tratamiento de la infección por VIH/SIDA ${ }^{13}$, que, según su mecanismo de acción, se clasifican en inhibidores nucleosídicos de la enzima transcriptasa reversa (INTR), inhibidores de la enzima transcriptasa reversa no nucleó- sidos (INNTR), inhibidores de la enzima proteasa (IP), inhibidores de la enzima integrasa (II) e inhibidores de la entrada, que incluye tanto a los bloqueadores del co-receptor CCR5 como a los bloqueadores de la fusión VIH-CD4. Los IP son quienes presentan un mayor número de efectos adversos severos ${ }^{14}$. Las reacciones adversas frecuentemente observadas durante las primeras semanas o meses de tratamiento incluyen reacciones hematológicas, dérmicas, gastrointestinales, toxicidad hepática, y alteraciones del sistema nervioso central; mientras que en las reacciones tardías incluyen alteraciones en sistema nervioso periférico y alteraciones metabólicas -trastornos en perfil lipídico, desarrollo de intolerancia a la glucosa, diabetes mellitus, redistribución del tejido graso, aparición de osteopenia y osteoporosis, entre otras ${ }^{14,15}$.

Estos antecedentes fundamentan la necesidad de realizar un seguimiento fármaco-terapéutico ambulatorio en pacientes que reciban TARV, de manera de detectar precozmente los problemas farmacológicos surgidos del tratamiento, permitiendo caracterizarlos y manejarlos adecuadamente para optimizar su terapia farmacológica.

\section{Pacientes y Métodos}

El seguimiento fármaco-terapéutico a pacientes en TARV se realizó en la Policlínica de Inmunología del Hospital Carlos Van Buren de Valparaíso (HCVB) durante 125 días (enero- mayo 2010). El protocolo utilizado fue previamente aprobado por el Comité de Ética del HCVB. 
Se incluyeron pacientes de ambos géneros, mayores de 18 años, en TARV, con controles clínicos periódicos, y que aceptaron participar en este estudio. Los criterios de exclusión fueron: embarazo, deseo de abandonar el seguimiento fármaco-terapéutico y la no asistencia a controles médicos.

El seguimiento farmacológico consistió en la determinación de parámetros clínicos periódicos que permitieran evaluar la eficacia y eventual toxicidad de la TARV prescrita. Se determinaron los problemas relacionados con la medicación (PRM) y se clasificaron en necesidad, efectividad, seguridad (toxicidad) o adherencia ${ }^{16}$.

La detección de toxicidad (PRM de seguridad) ocasionada por los anti-retrovirales, se realizó mediante los parámetros establecidos en la Guía Clínica vigen$\mathrm{te}^{14}$. Se registró en forma periódica los valores de los parámetros clínicos y, ante la sospecha de una reacción adversa, se evaluó la causalidad mediante el Algoritmo de Naranjo ${ }^{17,18}$ (Tabla 1) clasificándose como: definitiva, probable o posible. Posteriormente se determinó el grado de toxicidad, de acuerdo a lo establecido en la Guía Clínica para el Manejo de Pacientes con VIH/ SIDA $^{14}$ (Tabla 2).

Los valores de los parámetros clínicos se analizaron utilizando el programa Stata 9.0, determinándose sus medianas y observando las correlaciones parciales entre la variable dependiente y la variable independiente mediante la operación pcorr.

\section{Resultados}

Sesenta y siete pacientes cumplieron con los criterios de inclusión previamente establecidos (correspondiendo a 59,3\% de los pacientes con control periódico en la Policlínica de Inmunología del HCVB). Sólo un paciente abandonó el seguimiento por no presentar registro de retiro de medicamentos en un período mayor a los seis meses. La caracterización demográfica y clínica de los pacientes se incluye en la Tabla 3 y Tabla 4, respectivamente.

Todos los pacientes estudiados presentaron algún problema de farmacoterapia durante el estudio; 31 de ellos (47\%) presentaron un PRM, 14 (21,2\%) presentaron dos PRM, 8 (12,1\%) presentaron tres PRM y los 13 restante presentaron 4 PRM.

En la monitorización fármaco-terapéutica de los TARV de los pacientes seleccionados, se detectaron 99 problemas de terapéutica. Estos problemas en 49,5\% de los casos $(n=49)$ involucraron reacciones adversas (PRM de toxicidad), en $41,4 \%(n=41)$ estuvieron relacionados con la necesidad de incorporar algún fármaco para mejorar su adherencia farmacológica o manejo de sus efectos laterales (PRM de necesidad) y 9,1 \% $(n=9)$ debido a la falta de adherencia parcial a la terapia por parte del paciente (PRM de efectividad).

De los 41 PRM de necesidad, el 65,9\% se generó por la falta de un hipolipemiante en el tratamiento prescrito; 21,9\% requirió un protector de la mucosa gástrica; a

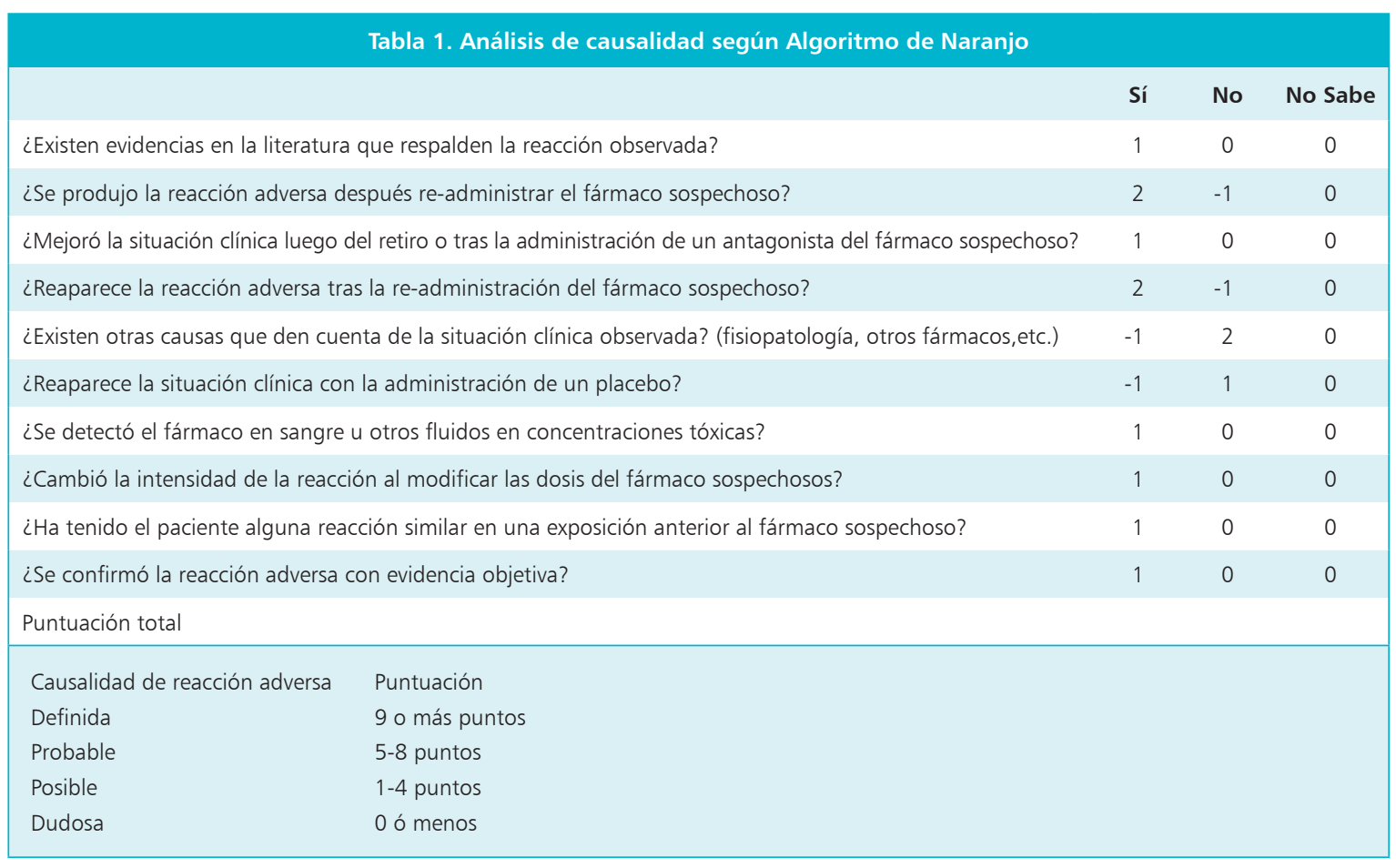


Tabla 2. Evaluación de toxicidades según grados de gravedad

\begin{tabular}{|c|c|c|c|c|}
\hline $\begin{array}{l}\text { Graduación de las principales } \\
\text { toxicidades }\end{array}$ & Grado 1 & Grado 2 & Grado 3 & Grado 4 \\
\hline Hematocrito (\%) & $28,5-31,4$ & $24-28,4$ & $19,5-23,9$ & $<19,5$ \\
\hline Hemoglobina (grs/\%) & $9,5-11$ & $8,0-9,4$ & $6,5-7,9$ & $<6,5$ \\
\hline Leucocitos $/ \mathrm{mm}^{3}$ & $2.500-3.999$ & $1.000-2.499$ & $800-999$ & $<800$ \\
\hline Recuento de neutrófilos / mm³ & $1.000-1.499$ & $750-999$ & $500-749$ & $<500$ \\
\hline Recuento de plaquetas $/ \mathrm{mm}^{3}$ & $75.000-99.000$ & $50.000-74.999$ & $20.000-49.999$ & $<20.000$ o petequias \\
\hline Hiperglicemia (mg/\%) & $116-160$ & $161-250$ & $251-500$ & $>500$ o cetoacidosis \\
\hline Hipertrigliceridemia (mg/\%) & $250-400$ & $401-750$ & $751-1.250$ & $>1.250$ \\
\hline Acidosis metabólica $\left(\mathrm{HCO}_{3}: \mathrm{mEq} / \mathrm{lt}\right)$ & $19-21$ & $15-18$ & $10-14$ & $<10$ \\
\hline Creatininemia (x límite máx normal) & $1,1-1,5$ & $1,6-3,0$ & $3,1-6,0$ & $>6$ o diálisis \\
\hline Bilirrubinemia (x límite máx normal) & $1,1-1,5$ & $1,6-2,5$ & $2,6-5,0$ & $>5$ \\
\hline GOT (x límite máx normal) & $1,25-2,5$ & $2,6-5,0$ & $5,1-10,0$ & $>10$ \\
\hline GPT (x límite máx normal) & $1,25-2,5$ & $2,6-5,0$ & $5,1-10,0$ & $>10$ \\
\hline GGT (x límite máx normal) & $1,25-2,5$ & $2,6-5,0$ & $5,1-10,0$ & $>10$ \\
\hline Fosfatasas alcalinas (x lím máx n) & $1,25-2,5$ & $2,6-5,0$ & $5,1-10,0$ & $>10$ \\
\hline Lipasemia (x límite máx normal) & $1,1-1,39$ & $1,4-2,09$ & $2,1-5,0$ & $>5$ o pancreatitis \\
\hline Amilasemia (x límite máx normal) & $1,1-1,39$ & $1,4-2,09$ & $2,1-5,0$ & $>5$ o pancreatitis \\
\hline Vómitos & Aislados & Frecuentes & Hipotensión o hidratación ev & Hipotensión grave y/o hospitalización \\
\hline Diarrea & $3-4$ x día & $5-7$ y/o nocturna & > 7, hipotensión, hidratación ev & Hipotensión grave y/o hospitalización \\
\hline Litiasis renal & \multicolumn{4}{|c|}{ Grado 4: Hematuria grave y/o insuficiencia renal obstructiva } \\
\hline Polineuropatía & Leve & Requiere tratamiento & Interfiere con el sueño & Limita la marcha \\
\hline Alteración del SNC & Ansiedad o depresión leve & Moderada & Grave, requiere asistencia & Psicosis aguda y/o hospitalización \\
\hline Alergia & Rash leve y/o prurito & $\begin{array}{c}\text { Máculas o máculo-pápulas } \\
\text { difusas }\end{array}$ & Generalizadas & Anafilaxis, Stevens Johnson o exfoliación \\
\hline Hipersensibilidad a abacavir & \multicolumn{4}{|c|}{ Grado 4: Rash con fiebre y/o síntomas digestivos o respiratorios } \\
\hline
\end{tabular}

9,8\% requirió la administración de ácido fólinico para complementar el tratamiento de la anemia; y a 2,4\% necesitó reemplazar cotrimoxazol por dapsona, debido a antecedentes de exantema alérgico.

En relación a los PRM de toxicidad, se aplicó el Algoritmo de Naranjo a las 49 situaciones posibles de ser consideradas como reacciones adversas; $85 \%$ se clasificó "probable" y el 15\% restante como "posible" (Tabla 5).

Se observó que el aumento del nivel de triglicéridos varió según el esquema TARV elegido, siendo mayor con el esquema basado en 2 INTR + 1 IP (con un valor mediana de $301,5 \mathrm{mg} / \mathrm{dL}$ ); para el esquema 2 INTR + 1 INNTR se determinó un valor de 278,0 mg/dL. También se observó una diferencia en los niveles de colesterol total (231 mg/ dL vs $200 \mathrm{mg} / \mathrm{dL})$, y colesterol LDL (122,5 mg/dL v/s $102,0 \mathrm{mg} / \mathrm{dL}$ ) obtenidos entre ambos tratamientos. En la Tabla 6 se observa un resumen de las reacciones adversas detectadas, mencionándose el porcentaje de aumento de los parámetro evaluados por sobre su valor mediana.
Cabe destacar que 38 pacientes (58\%) debieron abandonar el tratamiento original, debido principalmente a toxicidad por fármacos (Figura 1). La TARV mas prescrita (59\%) fue la asociación de los INTR más INNTR, seguido por prescripción en 33\% de los pacientes, de la asociación de los INTR más IP (Figura 2).

En relación a los nueve casos de PRM de efectividad, éstos se atribuyeron a una falta de adherencia parcial al tratamiento por un período superior a 15 días.

Además, durante el desarrollo del seguimiento fármaco-terapéutico se detectaron 34 potenciales interacciones farmacológicas (Tabla 7), la mayoría de las cuales eran de tipo farmacocinético, es decir afectarían las concentraciones plasmáticas de algunos de los fármacos involucrados.

Finalmente, se determinó que existe una relación entre el número de PRM detectados con el número de medicamentos prescritos, siendo determinante para la presentación de PRM que el paciente consuma más de 6 fármacos en forma concomitante (Tabla 8). 
Tabla 3. Características demográficas de los pacientes $(n=66)$

\section{Características}

Frecuencia (\%)

Género

Hombres $\quad 89,4$

Edad (años)

Rango 10-19

Rango 20-29 16,7

Rango 30-39 28,8

Rango 40-49 28,8

Rango 50-59 21,2

Rango 60-69 $\quad 1,5$

$>$ de 70

3,0

Orientación sexual

Homosexual

59,1

Heterosexual

36,4

Bisexual

4,5

Nivel educacional

Educación básica incompleta $\quad 7,6$

Educación media incompleta $\quad 33,3$

Educación media completa $\quad 43,9$

Educación técnica completa $\quad 6,1$

Educación superior completa $\quad 9,1$

Ocupación

Cesante 18,2

Trabajo estable $\quad 54,5$

Trabajo esporádico $\quad 16,7$

Jubilado $\quad 4,5$

Estudiante 3

Dueña de casa
Tabla 4. Caracterización clínica de los pacientes $(n=66)$

\section{Característica}

Frecuencia (\%)

Etapa clínica del paciente

$\begin{array}{ll}\text { Etapa VIH ( A1 , A2, B1, B2) } & 24,2 \\ \text { Etapa SIDA (A3, B3, C1, C2, C3) } & 75,8\end{array}$

Recuentol de linfocitos T CD4*

$\begin{array}{ll}>350 \text { células } / \mathrm{mm}^{3} & 34,8 \\ 350-200 \text { células } / \mathrm{mm}^{3} & 36,4 \\ <200 \text { células } / \mathrm{mm}^{3} & 28,8\end{array}$

Nivel de carga viral*

$\begin{array}{ll}>10.000 \text { copias } / \mathrm{mL} . & 16,7 \\ 10.000-80 \text { copias } / \mathrm{mL} . & 13,6 \\ <80 \text { copias } / \mathrm{mL} . & 69,7\end{array}$

(*) valores de medianas.

Tabla 5. Clasificación y frecuencia de reacciones adversas según su grado de toxicidad $(n=49)$

\begin{tabular}{lcccc} 
Toxicidad detectada & Grado $\mathbf{1}$ & Grado 2 & Grado 3 & Grado 4 \\
\hline Hipertrigliceridemia & $40,8 \%$ & $6,1 \%$ & $4,3 \%$ & $4,3 \%$ \\
\hline Aumento de bilirrubinemia & $2 \%$ & $6,1 \%$ & $6,1 \%$ & -- \\
\hline Aumento de transaminasa glutámico oxalacética & $6,1 \%$ & -- & -- & -- \\
\hline Aumento de transaminasa glutámico-pirúvica & $2 \%$ & $2 \%$ & $2 \%$ & -- \\
\hline Aumento de fosfatasa alcalina & $6,1 \%$ & -- & -- & -- \\
\hline Diarrea & -- & $6,1 \%$ & -- & -- \\
\hline Alteración del sistema nervioso central & $2 \%$ & -- & -- & -- \\
\hline Alergia & $2 \%$ & -- & $2 \%$ & -- \\
\hline
\end{tabular}

\section{Tabla 6 Frecuencia de aparición de reacción adversa en pacientes con tratamiento anti-retroviral $(n=49)$}

\section{Toxicidad detectadas}

Aumento del valor de bilirrubinemia directa

Aumento del valor de bilirrubinemia total

Aumento de triglicéridos

Aumento de triglicéridos

Aumento de colesterol total

Aumento en el valor de fosfatasas

Aumento en el valor de transaminasa glutámico oxalacética

Aumento en el valor de transaminasa glutámico-pirúvica

Anemia

\section{Fármacos sospechosos}

Atazanavir / ritonavir
Atazanavir / ritonavir

Esquema 2 INTR $^{2}+1$ INNTR $^{3}$

Esquema $2 \mathrm{INTR}^{2}+1 \mathrm{IP}^{4}$

Esquema 2 INTR $^{2}+1 \mathrm{IP}^{4}$

Efavirenz

Efavirenz

Efavirenz

Combinación zidovudina + lamivudina
$\%$ Alteración por sobre el valor de la mediana

$96 \%$

$221 \%$

$39 \%$

$50,8 \%$

$15,8 \%$

$3,1 \%$

$6,6 \%$

$46,3 \%$

$6,3 \%$
Indicador de prevalencia: n pacientes con $\mathrm{RAM}^{1} / \mathbf{n}$ pacientes con tratamiento evaluado

$63,6 \% \quad(7 / 11)$

$63,6 \% \quad(7 / 11)$

$38,5 \% \quad$ (5/39)

$27,2 \% \quad(6 / 22)$

$27,2 \% \quad(6 / 22)$

$7,5 \% \quad(3 / 40)$

$7,5 \% \quad(3 / 40)$

$7,5 \% \quad(3 / 40)$

$6,3 \% \quad(4 / 63)$

'RAM: reacción adversa medicamentosa, ${ }^{2}$ INTR (inhibidores de la enzima transcriptasa reversa), ${ }^{3}$ INNTR (inhibidores de enzima transcriptasa reversa no nucleósidos), ${ }^{4}$ IP (inhibidores enzima proteasa). 
Tabla 7.- Interacciones farmacológicas potenciales detectadas en pacientes con tratamiento anti-retroviral $(n=34)$

\section{Fármacos interactuantes Efecto Potencial}

Lamivudina / cotrimoxazol

Lopinavir-ritonavir / gemfibrozilo

Atazanavir / omeprazol

Lopinavir-ritonavir /

sertralina

Atazanavir / fenitoina

Fosamprenavir / etravirina

Lopinavir-ritonavir / con tenofovir

Lopinavir asociado con ritonavir con abacavir virológico
Cotrimoxazol aumenta en $43 \%$ los niveles plasmáticos de lamivudina posiblemente por disminuir el aclaramiento rena

Fluconazol aumenta hasta en $74 \%$ los niveles plasmáticos de zidovudina por reducción de su metabolización

TARV ${ }^{1}$ reduce en $41 \%$ la biodisponibilidad y Cmáx $^{2}$ de gemfibrozilo. Posiblemente el mecanismo sea una reducción de la absorción de gemfibrozilo, tal vez debido a la modulación de algún transportador intestinal por parte de lopinavir/ritonavir

a solubilidad de atazanavir se reduce a medida que aumenta el pH. La administración de omeprazol reduce en en $70 \%$ los niveles plasmáticos de atazanavir

Dapsona puede inhibir el metabolismo hepático de zidovudina. Incremento del riesgo de toxicidad hematológica aditiva

Lopinavir/ritonavir puede aumentar los niveles plasmáticos de sertralina por inhibición de su metabolismo a nivel de CYP3A4 ${ }^{3}$

Fenitoína es un antiepiléptico inductor de CYP450, que au menta el metabolismo hepático de atazanavir disminuyendo su concentración plasmática y favoreciendo un probable fracaso

Pueden aumentar parámetros farmacocinéticos de fosamprenavir (biodisponibilidad, Cmáx², $\mathrm{Cmín}^{4}$ )

Existe un aumento del riesgo de nefrotoxicidad con la asociación de tenofovir e inhibidores de la proteasa. Podría deberse a una interacción a nivel de transportadores

Los niveles plasmáticos de abacavir se reducen en $32 \%$ en presencia de lopinavir con ritonavir Probablemente debido a un aumento de la glucuronidación de abacavir por parte de la asociación lopinavir y ritonavir

\section{Recomendación}

Potencial interacción que puede justificar ajuste de dosis. Monitorizar toxicidad de lamivudina

Moderada interacción que puede justificar ajuste de dosis para zidovudina. Monitorizar toxicidad de zidovudina

Moderada interacción que puede justificar dosis de gemfibrozilo

Frecuencia

10

No recomendada. Se sugiere reemplazar omeprazol

por anti- $\mathrm{H}_{2}$ (ranitidina) y administrarlo $2 \mathrm{~h}$ después de atazanavir

Moderada interacción que puede justificar dosis de

4 zidovudina. Monitorizar toxicidad de zidovudina

Potencial interacción que puede justificar ajuste de

dosis. Monitorizar toxicidad de sertralina

Potencial interacción puede justificar la prescripción de antiepilépticos no inductores enzimáticos. En cualquier caso, no debería emplearse atazanavir no potenciado

Moderada interacción que puede justificar dosis de fosamprenavir

Potencial interacción que requiere monitorizar función renal

'TARV: tratamiento anti-retroviral, ${ }^{2} \mathrm{C}$ máx: concentración plasmática máxima; ${ }^{3} \mathrm{CYP} 3 \mathrm{~A} 4$ : citocromo P450 (subfamilia $\left.3 \mathrm{~A} 4\right) .{ }^{4} \mathrm{Cmin}:$ concentración plasmática mínima,

Tabla 8. Aparición de PRM1 según factores del paciente (edad y género) y número de medicamentos concomitantes $(n=66)$

\section{Factor evaluado}

Edad del paciente

\section{Indicador evaluado}

$\mathrm{N}^{\circ}$ pac.del rango etáreo con PRM

$N^{\circ}$ pac. total en el rango de edad

Género del paciente

$N^{\circ}$ pac. de cada género con $\mathrm{PRM}^{1}$

$\mathrm{N}^{\circ}$ pac. total de cada género

$\mathrm{N}^{\circ}$ pac.por rango fármacos $\mathrm{C} / \mathrm{PRM}$

$N^{\circ}$ pac. total por rango fármacos

\section{Rangos evaluados (años)}

Rango 20-29

Rango 30-39

Rango 40-49

Rango 50-59

Rango 60-69

Rango $>70$

Pacientes masculinos

Pacientes femeninos

$N^{\circ}$ pacientes con 1-3 fármacos

No pacientes con 4- 6 fármacos

$N^{\circ}$ pacientes con $>6$ fármacos

\section{Valor del indicador (\%)}

1,45

2,2

2,3

2,1

1,0

1,5

2,1

1,9

1,4

2,2

3,4

\section{Relación encontrada}

No existe relación entre edad del paciente y aparición de PRM ${ }^{1}$ $(p>0,05)$

No existe relación entre genero del paciente y aparición de PRM

$(p>0,05)$

Pacientes con más de 6 fármacos concomitantes presentan mayor número de $\mathrm{PRM}^{1} \mathrm{~s}$ $(p<0,05)$

$\mathrm{PRM}^{1}$ : Problemas relacionados con la medicación. 


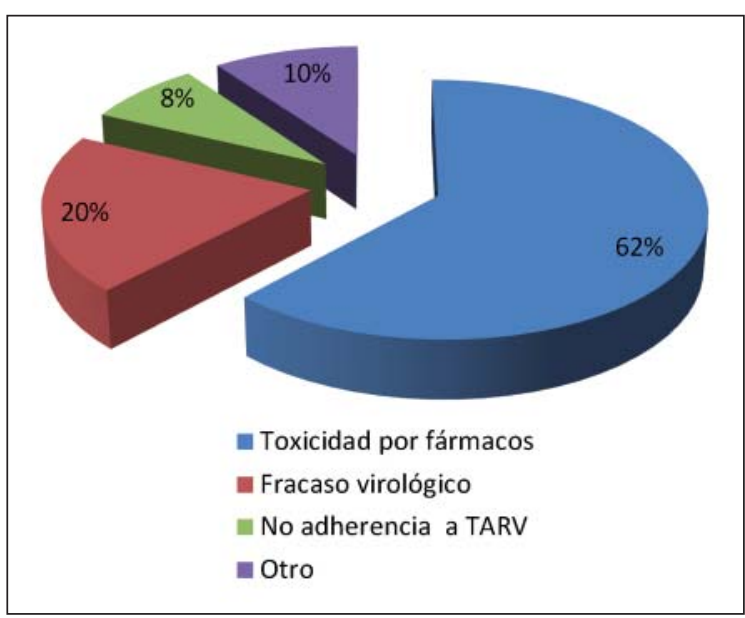

Figura 1. Distribución porcentual de los motivos de fracaso terapéutico $(n=38)$.

\section{Discusión}

En la caracterización de los pacientes en etapa SIDA se observó una alta tasa en el rango etáreo comprendido entre los 30 y 39 años, y con predominio del género masculino (89,2\%), concordando con lo encontrado en la literatura médica ${ }^{15,19}$.

El 58\% de los abandonos de tratamiento informados se debió a la toxicidad de fármacos, valor que presenta diferencia significativa con el informado en la Cohorte Chilena de SIDA del año 2007, lo que podría atribuirse en gran medida a una inadecuada administración de los medicamentos, que acentuarían la aparición de reacciones adversas y, por ende, de incumplimiento ${ }^{15,21}$.

En el análisis de la relación entre factores dependientes del paciente con los PRM detectados, sólo el número de fármacos utilizados presentó una asociación con significancia estadística, generalmente, aumentando la toxicidad de la terapia prescrita (reacciones adversas e interacciones). Esto se debería a que la mayor prescripción de fármacos aumenta la complejidad de la posología, aumentando a la vez la posibilidad de interacciones farmacológicas. De esta manera se hace necesario enfatizar la monitorización de los parámetros clínicos, especialmente, en pacientes con más de seis fármacos concomitantes (polifarmacia) de forma de detectar y tratar oportunamente los PRMs que se generen aumentando la eficacia, seguridad $\mathrm{y}$ adherencia del TARV ${ }^{22,23}$.

La reacción adversa más común fue la hipertrigliceridemia (55,5\%). Esto concuerda con estudios que han informado que la incidencia de hipertrigliceridemia oscila entre 5 y 90\%, dejando establecido que existen factores inmunológicos, virológicos y características propias de los pacientes, que inciden en la aparición de estas alteraciones. ${ }^{24,25,26,27,28,29}$. En este mismo sentido, la Cohorte

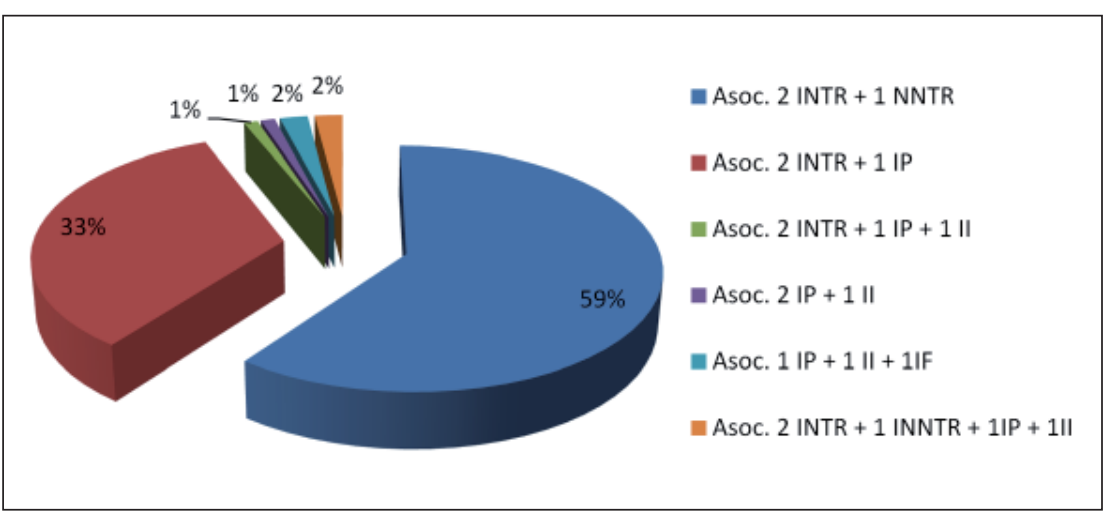

Figura 2. Distribución porcentual de tipos de tratamientos prescritos durante el estudio $(n=66)$. INTR: Inhibidores nucleosídicos de la enzima transcriptasa reversa; INNTR: Inhibidores de la enzima transcriptasa reversa no nucleósidos; IP: Inhibidores de la enzima proteasa; II: inhibidores de la enzima integrasa; IF: inhibidores de la entrada, que incluye tanto a los bloqueadores del co-receptor CCR5 como a los bloqueadores de la fusión VIH-CD4.

Chilena de SIDA ha informado que 3,2\% de las causas de cambio o suspensión del tratamiento por toxicidad se debe a hipertrigliceridemia grave (grado 3 a 4$)^{15}$.

En cuanto a las alteraciones en el perfil hepático, 16,6\% de los pacientes con tratamiento de atazanavir estudiados desarrolló hiperbilirrubinemia (Tabla 6), alteración que ha sido informada como toxicidad intrínseca frecuente para este anti-retroviral ${ }^{30,31}$. Además se observó aumento en los niveles de transaminasas producto del uso de efavirenz, que alcanzó grado 1 y 2 en nuestros pacientes. Sin embargo, es importante mencionar que existen informaciones sobre hepatotoxicidad grado 3 ó 4 asociada a este medicamento por lo que la monitorización de estos parámetros permitirá su detección precoz y la retirada del medicamento sospechoso de toxicidad ${ }^{10,32,33}$.

De las alteraciones en el hemograma, es posible observar que 6\% desarrolló una anemia leve; estos pacientes recibieron medicación concomitante con cotrimoxazol y la combinación zidovudina con lamivudina, concordando con lo publicado al señalar que la anemia es más frecuente en pacientes con tratamientos concomitantes de fármacos antagonistas de folatos y sulfonamidas, principalmente debido a la potenciación del efecto mielosupresor de cotrimoxazol $^{34,35}$.

Los problemas de efectividad determinados, correspondieron en $100 \%$ a la falta de adherencia parcial a la TARV prescrita.

Los PRMs detectados generaron acciones farmacéuticas, que fueron aceptadas en $100 \%$ (ya fuese por parte del equipo médico o de los pacientes afectados). Estas acciones abarcaron monitorización (de cargas virales, función renal, hemograma o perfil hepático), sugerencia de incorporación de fármacos (gemfibrozilo, ácido folínico, loperamida o loratadina), elaboración de cronograma de administración de medicamentos (evitando 
la administración simultánea de fármacos potencialmente interactuantes, o de manera de favorecer la absorción del fármaco) y reforzar los conocimientos de la enfermedad y/o tratamiento a manera de fomentar la adherencia a la TARV. Se registró una eficacia de 94,4\% en las acciones farmacéuticas realizadas, las que fueron evaluadas mediante la normalización de los parámetros clínicos previamente definidos. Cabe mencionar que en el caso de las acciones de tipo consejería y/o educativas no fue posible su evaluación debido a que son procesos que involucran cambios de hábitos de vida y/o comportamientos de riesgo que son difícil de modificar en el corto plazo.

En cuanto a las interacciones detectadas es necesario mencionar que algunos estudios les asignan una gran importancia debido a que reportan una alta prevalencia de ellas, como también por la gravedad que revisten ${ }^{10,11,36,37}$. En este estudio la mayoría de las interacciones observadas presentaron una recomendación de monitorizar; sin embargo, queda pendiente la determinación plasmática de los niveles de fármacos interactuantes. Otro aspecto del manejo farmacológico de este grupo de pacientes no abordados en el presente trabajo se refiere a determinar el consumo concomitante de suplementos nutricionales (especialmente sales de calcio), hierbas (tales como hierba de San Juan) y medicamentos de origen natural, que podrían afectar la farmacocinética de la TARV, y por ende su eficacia.

En suma, el uso de herramientas como el Algoritmo de Naranjo (para definir grado de relación causal de la alteración observada con la terapia farmacológica prescrita), asociada al análisis del nivel de toxicidad, permitió detectar en forma precoz los problemas asociados a la farmacoterapia de los pacientes. Estos problemas se relacionaron principalmente con la presentación de toxicidad intrínseca de los fármacos como también a las producidas por interacciones (principalmente de tipo farmacocinéticos), y su tratamiento oportuno se tradujo en un beneficio directo al paciente. Sin embargo, la determinación analítica de estas interacciones (a través de niveles plasmáticos de fármacos) como la aparición de otras interacciones queda pendiente a ser evaluado por futuros trabajos prospectivos.

\section{Resumen}

La adherencia al tratamiento anti-retroviral es un pilar fundamental en la reducción de la morbi-mortalidad de la infección por VIH/SIDA. Sin embargo, se ve dificultada por ser un tratamiento que involucra numerosos medicamentos administrados de forma crónica, con posibilidad de presentar reacciones adversas y/o interacciones. Se realizó un seguimiento farmacoterapéutico a 66 pacientes ambulatorios con tratamiento anti-retroviral del Hospital Carlos Van Buren. El 100\% de los pacientes presentó problemas relacionados con medicamentos, afectando en $46,1 \%$ a la seguridad de la terapia. La reacción adversa más frecuente fue hipertrigliceridemia, principalmente asociada al uso de dos inhibidores nucleosídico de la transcriptasa reversa con un inhibidor no nucleosídico de la transcriptasa reversa. Los resultados permiten concluir que la monitorización farmacológica de pacientes con tratamiento anti-retroviral identifica en forma precoz los problemas relacionados con medicamentos, favoreciendo la seguridad y eficacia de la terapia propuesta.

\section{Referencias bibliográficas}

1.- Palella F, Delaney K, Moorman A, Loveless M, Fuhrer J, Satten G, et al. Declining morbidity and mortality among patients with advanced human immunodeficiency virus infection. $\mathrm{N}$. Engl J Med 1998; 338: 853-60.

2.- Hogg R, Heath K, Yip B, Craib K, O'Shaughnessy M, Schechter M, et al. Improved survival among HIV-infected individuals following initiation of antiretroviral therapy. JAMA 1998; 279: 450-4.

3.- Lima V, Hogg R, Harrigan R, Moore D, Yip B, Wood E, et al. Continued improvement in survival among HIV- infected individuals with newer forms of highly active antiretroviral therapy. AIDS 2007; 6: 685-92.

4.- Alvis O, De Coll L, Chumbimune L, Díaz C, Díaz J, Reyes M. Factores asociados a la no adherencia al tratamiento antirretroviral de gran actividad en adultos infectados con el VIHSIDA. An Fac Med 2009; 70: 266-72.

5.- Torres A., Blasco B., González J., Martínez M.,
Ortiz de Urbina M., Jiménez V.et al., Adhesión al tratamiento antirretrovírico en pacientes VIH+. Farm Hosp. 2000. 24: 377-82.

6.- Knobel H, Escobar I, Polo R, Ortega L, Martín-Conde M T, Casado J L, et al. Recomendaciones GESIDA/SEFH/PNS para mejorar la adherencia al tratamiento antirretroviral en el año 2004 Enferm. Infecc. Microbiol Clin 2005; 23: 221-31.

7.- Organización Panamericana de la Salud. Tratamiento antirretroviral de la infección por el VIH en adultos y adolescentes en Latinoamérica y el Caribe: en la ruta hacia el acceso universal. 2008 visitada en http://www.paho.org el 30 de diciembre de 2011.

8.- Cabrera S, Valverde M P, García M J, Sánchez A, González M C. Intervención farmacéutica en el seguimiento de la terapia antirretroviral. An R Acad Nac Farm 2009; 75: 43-62.

9.- García B. Cumplimiento del tratamiento antirretroviral: Tendón de Aquiles de la nueva terapia de combinación. Noticias fármaco- terapéuticas 1997; 18: 1-4.

10.- Grimes R L, Lewis S T. Frequency of medical history items, drug interactions, an lifestyle characteristics that may interfere with antiretroviral medications. HIV Clin Trials 2002; 3: 161-7.

11.- Kashuba A. Drug-drug interactions and the pharmacotherapy of HIV infection. Top HIV Med 2005; 13: 64-9.

12.- Astuvilca J, Arce Y, Sotelo R, Quispe J, Guillén R, Peralta L et al. Incidencia y factores asociados con las reacciones adversas del tratamiento antirretroviral inicial en pacientes con VIH. Rev. Perú Med Exp Salud Publica 2007; 24: 218.

13.- Instituto Salud Pública. Sistema de Consulta de Productos Registrados. 2011 visitado en http://200.68.11.21/RegistrosISP/fiMenu.asp el 30 de diciembre de 2011.

14.- Ministerio de Salud (MINSAL). Guía Clínica Síndrome de Inmunodeficiencia Adquirida VIH/ SIDA. Santiago. 2010.visitado en http://www. minsal.gob.cl el 30/12/2011. 
15.- Ministerio de Salud (MINSAL) Comisión Nacional del SIDA y Grupo SIDA Chile Evaluación de impacto del programa de acceso expandido a tratamiento antirretroviral. Cohorte chilena de SIDA. Diciembre 2007. visitado en http://www.minsal.gob.cl el 30 de diciembre de 2011.

16.- Tercer Consenso de Granada sobre Problemas Relacionados con Medicamentos (PRM) y Resultados Negativos Asociados a la Medicación (RNM). Ars Pharm 2007; 48: 5-17.

17.- Naranjo C, Busto U, Sellers E, Sandor P, Ruiz I, Roberts E, et al. A method for estimating the probability of adverse drug reactions Clin Pharmacol Ther 1981; 239-45.

18.- Holloway K, Green T. Comités de Farmacoterapia - Guía Práctica Organización Mundial de la Salud. Departamento de Medicamentos Esenciales y Política Farmacéutica Ginebra, Suiza; 2003: 64-77.

19.- Panel on Antiretroviral Guidelines for Adults and Adolescents. Guidelines for the use of antiretroviral agents in HIV-1-infected adults and adolescents. Department of Health and Human Services. 2011; 1-167. disponible en http://www.aidsinfo.nih.gov/ página visitada 20 de Diciembre de 2011.

20.- Ministerio de Salud (MINSAL). Evolución del VIH-SIDA V Región, 1987-2006. Departamento de Epidemiología, DIPLAS, Agosto 2008.

21.- Afani A, Beltrán C, Gallardo A M, Roessler P, Acevedo W, Vásquez P. Prevalencia de resistencia primaria en pacientes con infección reciente por VIH-1 en Chile. Rev Med Chile 2010; 138: 669-76.

22.- Wolff M, Álvarez P, Flores I, Northland R, Wolff C. Evolución de mortalidad y estado actual de una población infectada por VIH controlada en un centro multiprofesional. Rev Méd Chile 2006; 134: 581-8.

23.- Walensky R P, Paltiel A, Losina E, Mercincavage L, Schackman B, Sax P, et al. 2 million years of life saved: the survival benefit of AIDS therapy in the United States. Disponible en http://www.niaid.nih.gov Visitado el 3 de enero de 2012.

24.- Savès M, Raffi F, Capeau J, Rozenbaum W, Ragnaud JM, Perronne C, et al. Antiprotéases Cohorte (APROCO) Study Group. Factors related to lipodystrophy and metabolic alterations in patients with human immunodeficiency virus infection receiving highly active antiretroviral therapy. Clin Infect Dis 2002; 34: 1396-405.

25.- Heath K, Hogg R, Singer J, Chan K, O’Shaughnessy M, Montaner J. Antiretroviral treatment patterns and incident HIV-associated morphologic and lipid abnormalities in a population- based cohort. J Acquir Immune Defic Syndr 2002; 30: 440-7.

26.- Leclercq P, Blanc M. Metabolic abnormalities, lipodystrophy and cardiovascular risk in HIVinfected patients. Rev Prat 2006; 56: 987-94.

27.- Van der Valk M, Bisschop P H, Romijn J, Ackermans M T, Lange J, Endert E, et al. Lipodystrophy in HIV-1-positive patients is associated with insulin resistance in multiple metabolic pathways. AIDS 2001; 15: 2093-100.

28.- Diehl L, Dias J, Paes A, García L, Cinagawa E, et al. Prevalência da lipodistrofia associada ao HIV em pacientes ambulatoriais brasileiros: Relação com síndrome metabólica e fatores de risco cardiovascular. Arq Brás Endrocrinol Metab 2008; 52-4.

29.- Morales M, Moreno M J, Abellán J, Rubio R.
Efectos secundarios graves derivados de las interacciones medicamentosas del tratamiento antirretroviral. Rev Clin Esp 2008; 208 : 557-60.

30.- Smith D E, Jeganathan S, Ray J. Atazanavir plasma concentrations vary significantly between patients and correlate with increased serum bilirubin concentrations. HIV Clin Trials 2006; 7: 34-8.

31.- Rodríguez-Novoa S, Martin-Carbonero L, Barreiro P, González-Pardo G, Jiménez-Nácher I, González-Lahoz J, et al. Genetic factors influencing atazanavir plasma concentrations and the risk of severe hyperbilirubinemia. AIDS 2007; 21: 41-6.

32.- Setzer B, Lebrecht D, Walker UA. Pyrimidine nucleoside depletion sensitizes to the mitochondrial hepatotoxicity of the reverse transcriptase inhibitor stavudine. Am J Pathol 2008; 172: 681-90.

33.- Schouten J T, Krambrink A, Ribaudo H J, Kmack A, Webb N, Shikuma C, et al. Substitution of nevirapine because of efavirenz toxicity in AIDS clinical trials group A5095. Clin Infect Dis 2010; 50: 787-91.

34.- Israel D S, Plaisance K I. Neutropenia in patients infected with human inmunodeficiency virus. Clin Pharm 1991; 10: 268-79.

35.- Meidani M, Rezaei F, Reza M, Avijgan M, Tayeri K. Prevalence, severity, and related factors of anemia in HIV/AIDS patients. J Res Med Sci 2012; 17: 138-42.

36.- Lee B, Safrin S. Interactions and toxicities of drugs used in patients with AIDS. Clin Infect Dis 1992; 14: 773-9.

37.- Jiménez V. Calidad Fármaco-terapéutica, Universidad de Valencia, Editorial Valencia 2006; pag. 620-3. 